\title{
TITLE:
}

\section{Electron acceleration by Z-mode and whistler-mode waves}

$\operatorname{AUTHOR}(S):$

Lee, K. H.; Omura, Y.; Lee, L. C.

\section{CITATION:}

Lee, K. H....[et al]. Electron acceleration by Z-mode and whistler-mode waves. Physics of Plasmas 2013, 20(11): 112901.

ISSUE DATE:

2013-11-11

URL:

http://hdl.handle.net/2433/179770

RIGHT:

(C) 2013 AIP Publishing LLC 


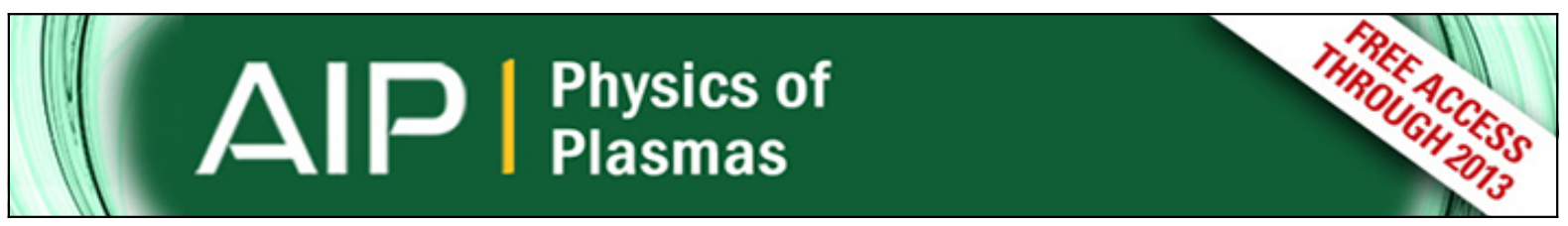

\section{Electron acceleration by Z-mode and whistler-mode waves}

K. H. Lee, Y. Omura, and L. C. Lee

Citation: Physics of Plasmas (1994-present) 20, 112901 (2013); doi: 10.1063/1.4829439

View online: http://dx.doi.org/10.1063/1.4829439

View Table of Contents: http://scitation.aip.org/content/aip/journal/pop/20/11?ver=pdfcov

Published by the AIP Publishing

AlP Re-register for Table of Content Alerts 


\title{
Electron acceleration by Z-mode and whistler-mode waves
}

\author{
K. H. Lee, ${ }^{1}$ Y. Omura, ${ }^{2, a)}$ and L. C. Lee $e^{1,3, a)}$ \\ ${ }^{1}$ Institute of Space Science, National Central University, Zhongli, Taiwan \\ ${ }^{2}$ Research Institute for Sustainable Humanosphere, Kyoto University, Uji, Kyoto, Japan \\ ${ }^{3}$ Institute of Earth Science, Academia Sinica, Nankang, Taiwan
}

(Received 29 August 2013; accepted 20 October 2013; published online 11 November 2013)

We carried out a series of particle simulations to study electron acceleration by Z-mode and whistler-mode waves generated by an electron ring distribution. The electron ring distribution leads to excitations of $\mathrm{X}$-mode waves mainly in the perpendicular direction, Z-mode waves in the perpendicular and parallel directions, and whistler-mode waves mainly in the parallel direction. The parallel Z- and whistler-mode waves can lead to an effective acceleration of ring electrons. The electron acceleration is mainly determined by the wave amplitude and phase velocity, which in turn is affected by the ratio of electron plasma to cyclotron frequencies. For the initial kinetic energy ranging from 100 to $500 \mathrm{keV}$, the peak energy of the accelerated electrons is found to reach 2-8 times the initial kinetic energy. We further study the acceleration process by test-particle calculations in which electrons interact with one, two, or four waves. The electron trajectories in the one-wave case are simple diffusion curves. In the multi-wave cases, electrons are accelerated simultaneously by counter-propagating waves and can have a higher final energy. (C) 2013 AIP Publishing LLC. [http://dx.doi.org/10.1063/1.4829439]

\section{INTRODUCTION}

Generation of energetic particles in space and laboratory plasmas is an important topic in plasma physics. Recently, electron acceleration and diffusion by whistler-mode chorus waves and electromagnetic ion cyclotron waves ${ }^{1-4}$ have been widely discussed. The phase difference between the electron gyrophase and wave phase plays a crucial role in this acceleration process. In our previous study, we showed that the electron ring distribution excites, through cyclotron maser process, ${ }^{5}$ the X-mode waves mainly in the perpendicular direction, Z-mode waves in the perpendicular and parallel directions, and whistler-mode waves mainly in the parallel direction. ${ }^{6,7}$ The parallel Z-mode waves lead to electron acceleration and formation of X-like momentum distribution. $^{7}$ The electron ring distributions can be produced in quasi-perpendicular shocks, ${ }^{8,9}$ by mirror reflection of energetic plasma beam in coronal flux loops ${ }^{10}$ and by injection of electron beam in the direction perpendicular to magnetic field. ${ }^{11}$

In this paper, we present the results of particle simulations and test-particle calculations. It is found that there exist three types of electron acceleration and diffusion in velocity space associated with Z-mode and whistler-mode waves. The strong acceleration is related to electrons resonant trapping by waves, and wave phase velocity, amplitude, and frequency play important roles.

\section{SIMULATION SETUP}

The 1D and 2D electromagnetic particle-in-cell simulation codes used in this paper are modified from KEMPO1. ${ }^{12}$ The 2D simulation box lies in the $\mathrm{x}-\mathrm{y}$ plane with periodic

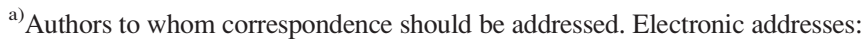
omura@rish.kyoto-u.ac.jp and loulee@jupiter.ss.ncu.edu.tw
}

boundaries. The 1D simulation box is along the $\mathrm{x}$-axis with periodic boundaries. In the initial condition, all particles are uniformly distributed in the simulation box with a constant ambient magnetic field $\mathbf{B}_{\mathbf{0}}=B_{0} \hat{x}$ for $2 \mathrm{D}$ and $\mathbf{B}_{0}=B_{0} \cos \theta_{B} \hat{x}+B_{0} \sin \theta_{B} \hat{y}$ with wave vector $\mathbf{k} \| \hat{x}$ for 1D. The protons are assumed to be cold and immobile. A ring distribution function in the cylindrical coordinates can be expressed as

$$
f_{r}=n_{r} a_{r} \exp \left[-\frac{\left(u_{\perp}-u_{r \perp}\right)^{2}+u_{\|}^{2}}{2(\Delta u)^{2}}\right],
$$

where $u_{\perp}$ and $u_{\|}$are momentum components perpendicular and parallel to ambient magnetic field $\mathbf{B}_{\mathbf{0}}$, respectively; $u_{r \perp}$ is the ring momentum and $a_{r}$ is a normalization constant. Here, the term "momentum" refers to "momentum per unit mass," $\mathbf{u}=\gamma \mathbf{v}$, where $\gamma$ is the Lorentz factor. The density of the ring distribution $n_{r}$ is set to $5 \%$ of total electron density. The momentum dispersion of energetic ring electrons is $\Delta u=0.025 c$, and the thermal momentum of background electrons is $0.05 c$, where $c$ is the speed of light. The ratio of electron plasma $\left(\omega_{p e}\right)$ to cyclotron $\left(\Omega_{c e}\right)$ frequencies $\alpha=\omega_{p e} / \Omega_{c e}$ is set to $0.33,1$, and 5 . The average initial kinetic energy, $\varepsilon_{0} \equiv\left(\gamma_{0}-1\right) m_{e} c^{2}$, of ring distribution is set to 100,200 , and $500 \mathrm{keV}$ in the simulation, corresponding to initial Lorentz factor $\gamma_{0} \equiv \sqrt{1+u_{r \perp}^{2} / c^{2}}=1.2,1.4$, and 2 , respectively, where $m_{e}$ is electron rest mass.

\section{EVOLUTION OF ENERGETIC ELECTRON DISTRIBUTION}

Fig. 1 shows energetic electron momentum distributions after the saturation of wave energy in 1D simulations with $\alpha=0.33$ and 1 at $\theta_{B}=0^{\circ}, 45^{\circ}$, and $90^{\circ}$. For $\theta_{B}=90^{\circ}$, waves tend to decelerate electrons along $u_{\perp}$. For $\theta_{B}=0^{\circ}$, the cases 

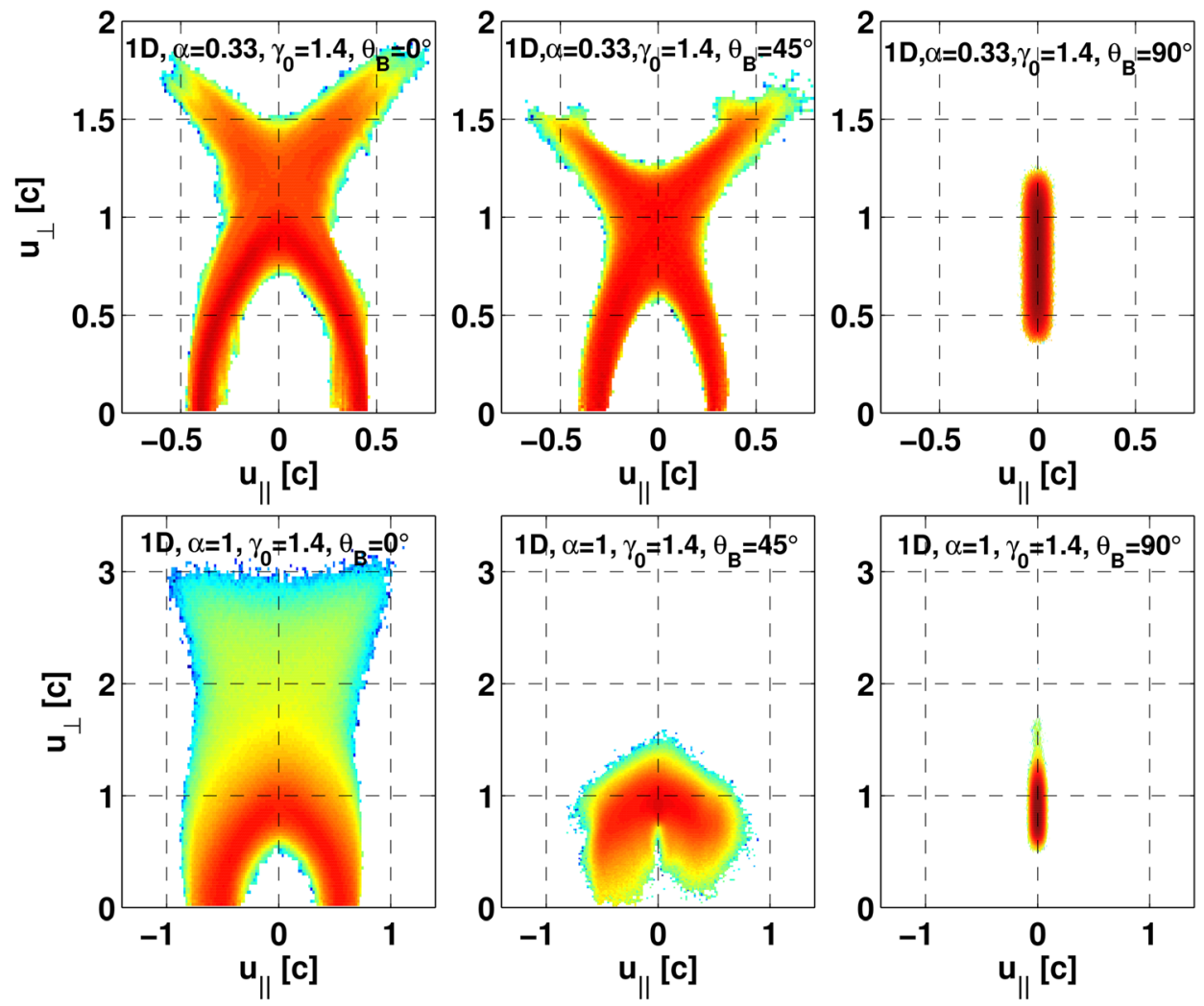

FIG. 1. Energetic electron distributions in $u_{\perp}-u_{\|}$momentum space after wave energy saturation of simulations for the $1 \mathrm{D}$ cases with $\alpha=0.33$ (top panels) and 1 (bottom panels) at $\theta_{B}=0^{\circ}, 45^{\circ}$, and $90^{\circ}$. The cases with $\theta_{B}=0^{\circ}$ show strong acceleration.

show strong acceleration of electrons, and the ring distribution evolves into an X-like pattern. For in-between cases, the acceleration or diffusion is stronger than that of $\theta_{B}=90^{\circ}$ but weaker than that of $\theta_{B}=0^{\circ}$. The acceleration process is clearly dominated by the waves propagating in the parallel direction.

We then compare the 1D and 2D simulation results. Note that in the following we assume parallel propagation of waves $\left(\theta_{B}=0^{\circ}\right)$ in the $1 \mathrm{D}$ simulations. Fig. 2 shows the energetic electron distributions in $u_{\perp}-u_{\|}$momentum space after the saturation of wave energy in the $1 \mathrm{D}$ and 2D simulations.

For $\alpha=0.1$, the acceleration is weak. Most electrons are decelerated in the perpendicular direction in the 2D cases, and the waves are too weak to diffuse electrons effectively in the $1 \mathrm{D}$ cases.

For $\alpha=0.33$, the $1 \mathrm{D}$ and $2 \mathrm{D}$ simulation results are similar. We can see clear X-like pattern and strong acceleration of electrons in both 1D and 2D cases. The peak energy of accelerated electrons can reach $3 \sim 4$ times the initial kinetic energy as shown in Fig. 3(a).

For $\alpha=1$, the electron acceleration is much stronger than other cases, but the X-like pattern is unclear. In the 2D cases, the waves with oblique and perpendicular propagations lead to a more diffusive pattern of electron distributions. The peak energy of accelerated electrons can reach 5-6 times the initial kinetic energy as shown in Fig. 3(b). For $\varepsilon_{0}=500 \mathrm{keV}$, the ring electrons can be accelerated to a peak energy $2.75 \mathrm{MeV}$ in the $1 \mathrm{D}$ case and $3 \mathrm{MeV}$ in the $2 \mathrm{D}$ case; for $\varepsilon_{0}=200 \mathrm{keV}$, electrons can be accelerated to $1.25 \mathrm{MeV}$ for $1 \mathrm{D}$ and $1.4 \mathrm{MeV}$ for $2 \mathrm{D}$; for $\varepsilon_{0}=100 \mathrm{keV}$, electrons can be accelerated to $800 \mathrm{keV}$ for 1D and $850 \mathrm{keV}$ for $2 \mathrm{D}$

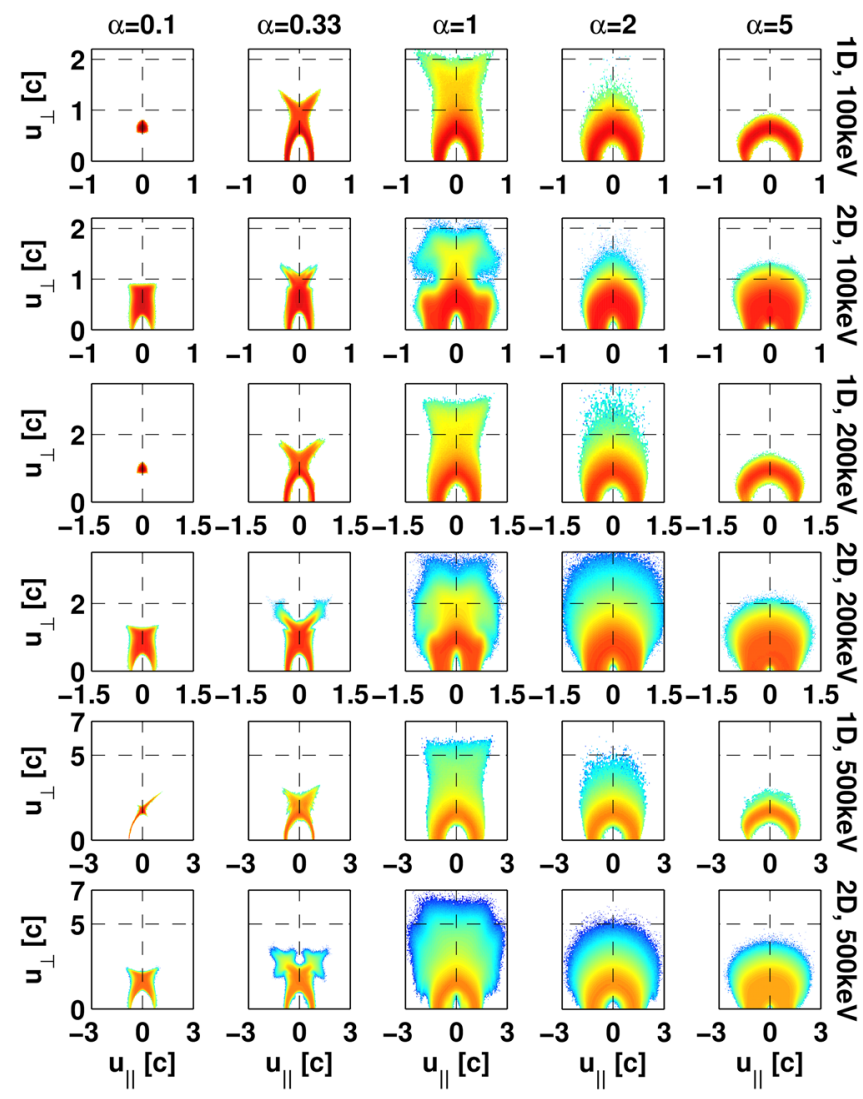

FIG. 2. Energetic electron distributions in $u_{\perp}-u_{\|}$momentum space after wave energy saturation of simulations for the $1 \mathrm{D}$ and $2 \mathrm{D}$ cases with $\alpha=0.1$, $0.33,1,2$, and 5 . 

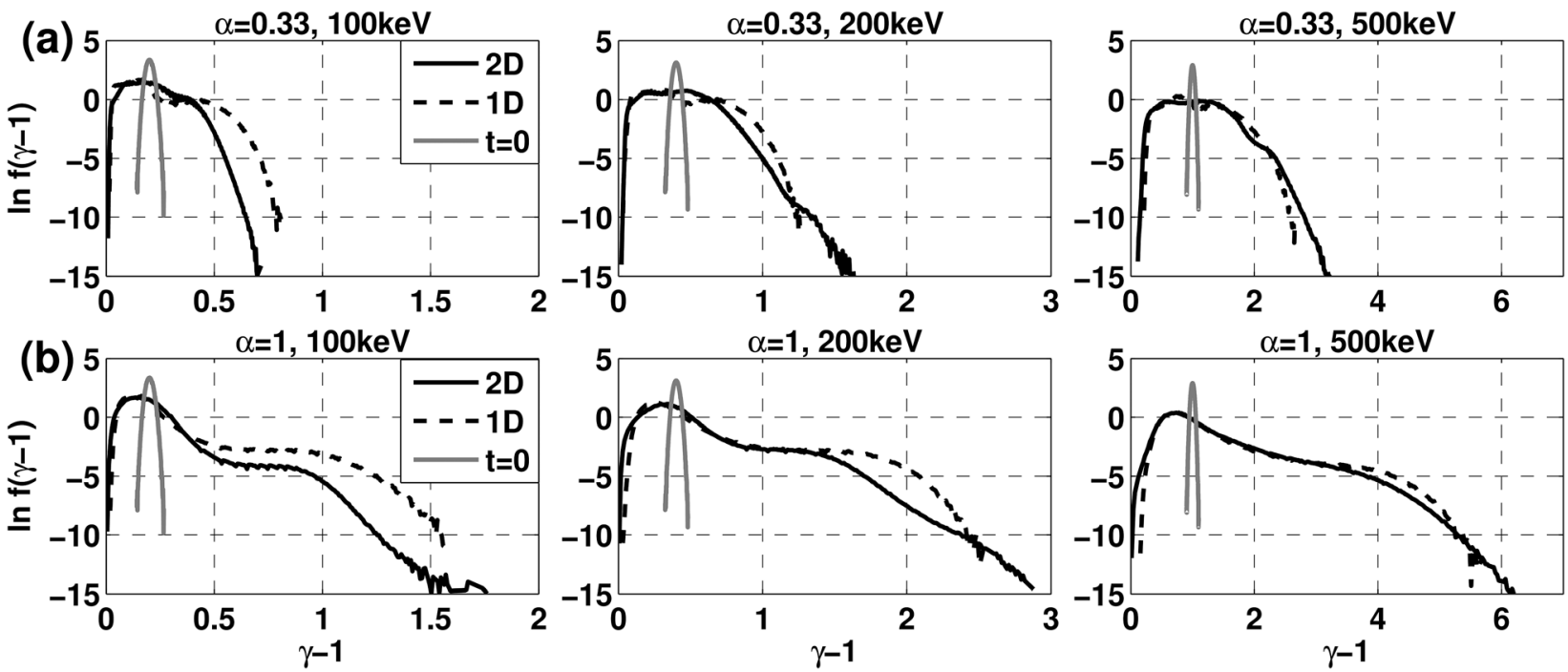

FIG. 3. Energetic electron distributions as a function of $(\gamma-1)$ for the $1 \mathrm{D}$ and $2 \mathrm{D}$ cases with $\alpha=0.33$ and 1 and initial kinetic energies $\varepsilon_{0}=100$, 200, and $500 \mathrm{keV}$.

For $\alpha=2$, the X-like pattern vanishes. Both 1D and 2D cases show shell-like distributions with hot tails in the perpendicular direction. For $\alpha=5$, the initial electron ring distribution evolves into a shell shape, showing pitch-angle scattering with little kinetic energy variation. These shelllike distribution could then trigger cyclotron maser instability for wave excitations in the perpendicular direction in 2D simulations, leading to a more diffusive pattern.

Table I shows the peak kinetic energies of accelerated electrons in 1D (2D) simulations as a function of $\alpha=\omega_{p e} / \Omega_{c e}$ and the initial kinetic energy $\varepsilon_{0}$. The maximum ratio of the peak to initial kinetic energies is 8 (8.5) and occurs in the case with $\alpha=1$ and $\varepsilon_{0}=100 \mathrm{keV}$. The maximum energy gain is $2250 \mathrm{keV}(2600 \mathrm{keV})$, and it occurs in the case with $\alpha=1$ and $\varepsilon_{0}=500 \mathrm{keV}$.

\section{WAVE EXCITATIONS BY ELECTRON RING DISTRIBUTION}

We examine wave excitations in the simulations and compare the results with kinetic theory. In the theoretical calculation, the energetic electron ring distribution is assumed to be a delta function, and the Doppler effect in the resonance condition vanishes. The excited waves are assumed to be at the first harmonic $\omega=\Omega_{c e} / \gamma_{0}$. Fig. 4 shows cold plasma dispersion diagrams of $\mathrm{Z}$ and whistler modes as

TABLE I. Peak kinetic energies of accelerated electrons in 1D (2D) simulations as a function of $\alpha=\omega_{p e} / \Omega_{c e}$ and the initial kinetic energy $\varepsilon_{0}$.

\begin{tabular}{llll}
\hline$\varepsilon_{\alpha=0.33}$ & $100 \mathrm{keV}$ & $200 \mathrm{keV}$ & $500 \mathrm{keV}$ \\
& & & \\
$\alpha=1.0$ & $400 \mathrm{keV}$ & $600 \mathrm{keV}$ & $1300 \mathrm{keV}$ \\
& $(350 \mathrm{keV})$ & $(800 \mathrm{keV})$ & $(1600 \mathrm{keV})$ \\
$\alpha=5.0$ & $800 \mathrm{keV}$ & $1250 \mathrm{keV}$ & $2750 \mathrm{keV}$ \\
& $(850 \mathrm{keV})$ & $(1400 \mathrm{keV})$ & $(3100 \mathrm{keV})$ \\
& $200 \mathrm{keV}$ & $400 \mathrm{keV}$ & $1000 \mathrm{keV}$ \\
& $(400 \mathrm{keV})$ & $(800 \mathrm{keV})$ & $(2000 \mathrm{keV})$ \\
\hline \hline
\end{tabular}

well as $\mathrm{X}$ and $\mathrm{O}$ modes at arbitrary propagation angles $\theta_{B}$ for $\alpha<1, \alpha=1$, and $\alpha>1$. We will concentrate on the $\mathrm{Z}$ and whistler modes.

In Fig. 4(a), for $\alpha<1 / \gamma_{0}<1 \quad\left(\omega_{p e}<\omega=\Omega_{c e} / \gamma_{0}\right.$ $<\Omega_{c e}$ ), the excited wave has frequency $\omega_{p e}<\omega<\Omega_{c e}$ and is the $\mathrm{Z}$ mode with $v_{p h}<c$ (red). For $1 / \gamma_{0}<\alpha<1$ $\left(\omega=\Omega_{c e} / \gamma_{0}<\omega_{p e}<\Omega_{c e}\right)$, the whistler mode with $\omega<\omega_{p e}$ is excited in the parallel and oblique directions (blue). The $\mathrm{Z}$ mode with $\omega<\omega_{p e}$ and $v_{p h}>c$ can be excited in the perpendicular and oblique directions if $\omega_{L}<\omega$ (green), where $\omega_{L}$ is the L-mode cut-off frequency. For $\alpha<1$ and $\theta_{B}=0^{\circ}$, the $\mathrm{Z}$ and whistler modes share the same branches in the $\omega-k$ dispersion diagram, and the separation point is $\omega=\omega_{p e}$.

In Figs. 4(b) and 4(c), for $1 / \gamma_{0}<1 \leq \alpha\left(\omega=\Omega_{c e} / \gamma_{0}\right.$ $<\Omega_{c e} \leq \omega_{p e}$ ), the whistler mode in the parallel and oblique directions can be excited (blue). The $\mathrm{Z}$ mode with $v_{p h}>c$ can be excited if $\omega_{L}<\omega$ (green). Since, $\omega=\Omega_{c e} / \gamma_{0}$ and $1 / \gamma_{0}$ is always smaller than unity, without Doppler effect, the $\mathrm{Z}$ mode with $\omega \geq \Omega_{c e}$ (red) cannot be excited.

According to the theoretical wave dispersion relations in Fig. 4, we can identify the wave modes observed in the simulation. Fig. 5 shows the wave dispersion diagrams of $\alpha=0.33,1$, and 5 obtained from $1 \mathrm{D}$ simulations. The $\mathrm{Z}$ mode dominates in the case of $\alpha=0.33$, and the whistler mode dominates in the cases of $\alpha=1$ and 5 .

Fig. 6 shows the theoretical linear growth rate (see, e.g., Ref. 8), wave number and phase velocity as a function of $\alpha=\omega_{p e} / \Omega_{c e}$ at $\theta_{B}=0^{\circ}$. The waves excited at $\omega=\Omega_{c e} / \gamma_{0}$ are the $\mathrm{Z}$ mode if $\alpha<1 / \gamma_{0}$ and are the whistler mode if $\alpha>1 / \gamma_{0}$. In Fig. 6(a), the growth rate of parallel whistler mode is higher than that of the parallel $\mathrm{Z}$ mode. The parallel $\mathrm{Z}$ mode is less active if $\alpha \leq 0.2$, and the acceleration of electrons is weak in the simulations with $\alpha \leq 0.2$ as discussed earlier. As $\alpha$ increases, the wave number of the excited waves increases as shown in Fig. 6(b), and the phase velocity decreases as shown in Fig. 6(c). The phase velocity is $v_{p h}>0.5 c$ for the parallel $\mathrm{Z}$ mode and is $v_{p h}<0.5 c$ for the whistler mode. For $\alpha \gg 1$, the phase velocities of amplified waves are much less than the speed of light as shown in 

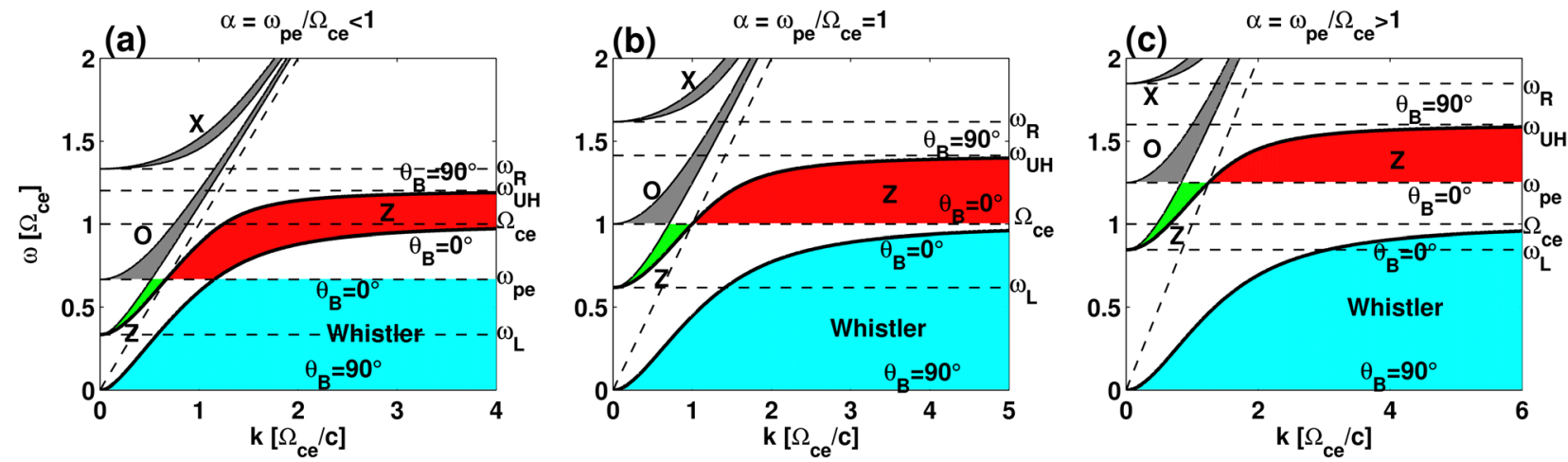

FIG. 4. Cold plasma dispersion relations of $\mathrm{Z}$ and whistler modes as well as $\mathrm{X}$ and $\mathrm{O}$ modes at arbitrary propagation angles $\theta_{B}$ for (a) $\alpha<1$ and (b) $\alpha=1$ and (c) $\alpha>1$. The X-mode cut-off, upper hybrid (Z-mode resonant) and L-mode cut-off frequencies are $\omega_{R}, \omega_{U H}$, and $\omega_{L}$, respectively.

Fig. 6(c). For electromagnetic waves, if the wave energy is the same, a lower phase velocity leads to a weaker wave electric field according to Faraday's Law $E_{w} / c B_{w}=\omega / c k \ll 1$. Hence $E_{w} \ll c B_{w}$, and the acceleration by wave electric field is weak when $\alpha \gg 1$ as the simulation cases of $\alpha=5$ shown in Fig. 2. For $\alpha \ll 1$, the growth rate of unstable waves is relatively low as shown in Fig. 6(a), and the wave amplitude is
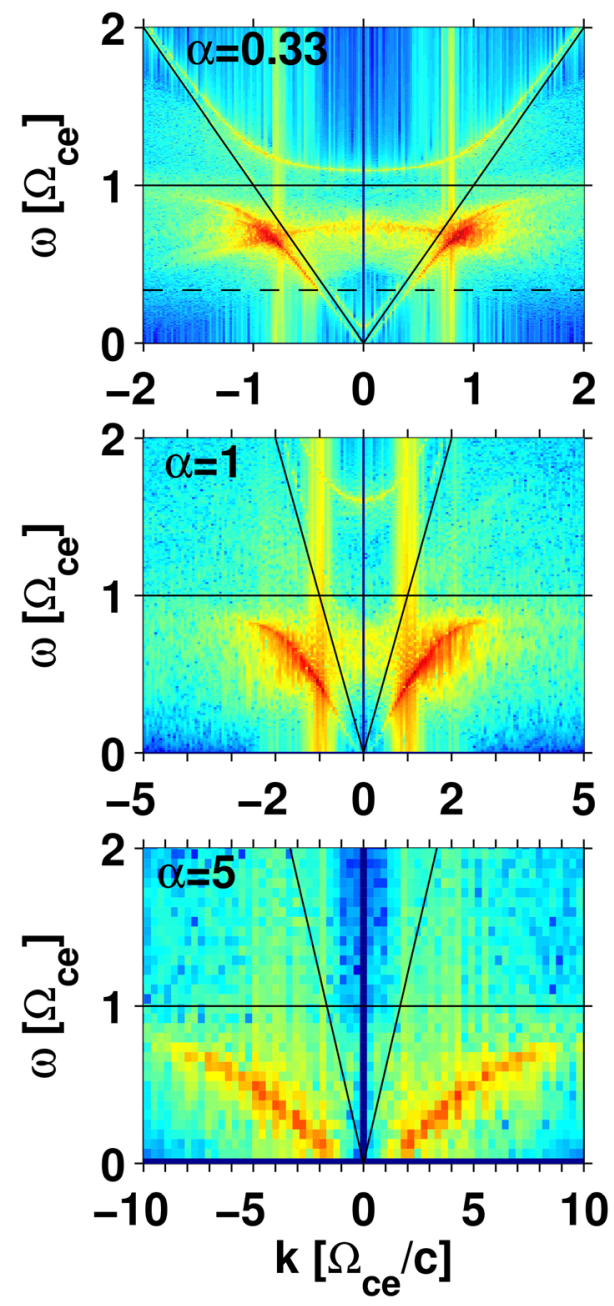

FIG. 5. Wave dispersion diagrams of $\alpha=0.33,1$, and 5 obtained from 1D simulations with $\theta_{B}=0^{\circ}$. The $\mathrm{Z}$ mode dominates in the case of $\alpha=0.33$, and the whistler mode dominates in the cases of $\alpha=1$ and 5. The dashed horizontal line indicates the plasma frequency. expected to be small. Hence, the acceleration is also weak when $\alpha \ll 1$ as the simulation cases of $\alpha=0.1$ shown in Fig. 2.

In Fig. 7, we compare the energetic electron distributions obtained from the 1D simulations with diffusion curves. The diffusion curve is determined by the conservation of kinetic energy in the frame of reference moving with $v_{p h}$

$$
v_{\|}^{\prime 2}+v_{\perp}^{\prime 2}=\text { const. }
$$

where

$$
v_{\|}^{\prime}=\frac{v_{\|}-v_{p h}}{1-v_{\|} v_{p h} / c^{2}}
$$
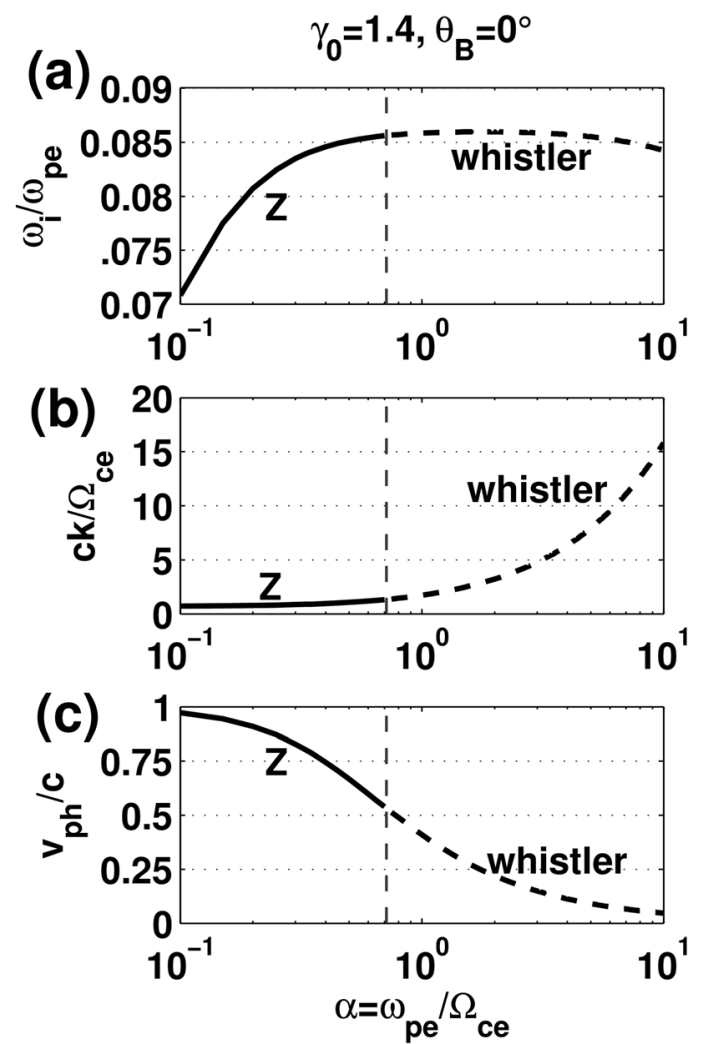

FIG. 6. (a) Theoretical linear growth rate, (b) wave number $k$ and (c) phase velocity $v_{p h}$ as functions of $\alpha=\omega_{p e} / \Omega_{c e}$ for $\gamma_{0}=1.4$ and $\theta_{B}=0^{\circ}$. The corresponding wave real frequency is $\omega_{r}=\Omega_{c e} / \gamma_{0} \approx 0.71 \Omega_{c e}$. The vertical dashed line corresponds to $\alpha=1 / \gamma_{0} \approx 0.714$. 

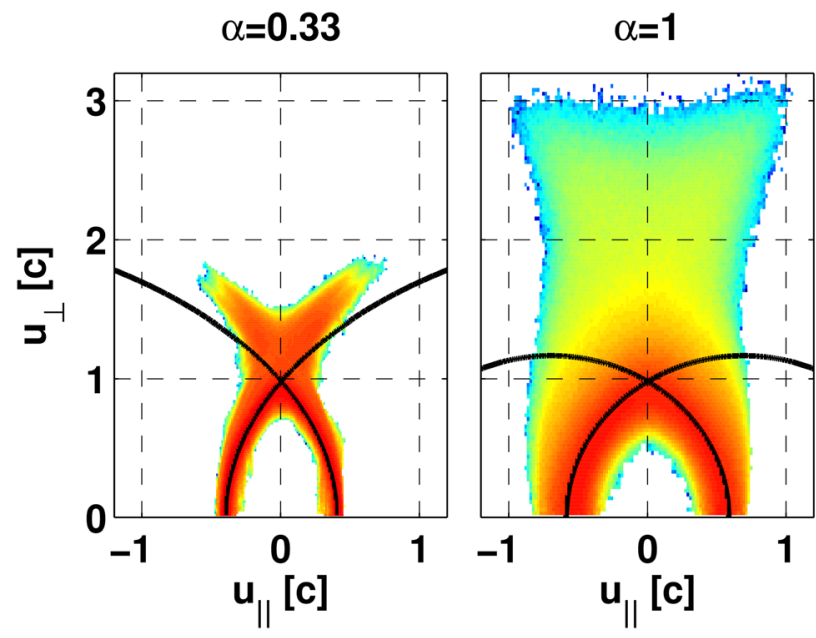

$$
v_{\perp}^{\prime}=v_{\perp} \frac{\sqrt{1-v_{p h}^{2} / c^{2}}}{1-v_{\|} v_{p h} / c^{2}} .
$$

Since $v_{p h}$ of intensive waves in each case of simulations are almost the same as shown in Fig. 5, the diffusion curves of these waves are also similar. The formation of X-like electron distribution in the case with $\alpha=0.33$ roughly coincides with the diffusion curves of the parallel Z-mode waves with high phase velocity and can be explained by one-wave resonant diffusion. ${ }^{7,13}$ In the case with $\alpha=5$, the initial electron ring distribution evolves into a shell shape, which is essentially the same as the corresponding diffusion curves. However, the acceleration of electrons by the whistler mode with medium phase velocity in the case with $\alpha=1$ cannot be explained by the diffusion curves.

\section{TEST-PARTICLE CALCULATION}

In our previous study, ${ }^{7}$ the test-particle results of $\alpha=0.33$ and $\gamma_{0}=1.4$ show that the kinetic energy increment in the one-wave case is comparable to that in the twowave case. For the $\mathrm{Z}$ mode with high phase velocity, onewave resonant diffusion can lead to strong acceleration of energetic electrons. However, for whistler-mode waves with medium phase velocity, the acceleration of electrons by counter-propagating waves is important.

To further analyze the acceleration process, we conduct a series of test-particle calculations. The equation of electron motion is

$$
m_{e} \frac{d(\gamma \mathbf{v})}{d t}=-e\left[\mathbf{E}_{f}+\mathbf{E}_{b}+\mathbf{v} \times\left(\mathbf{B}_{0}+\mathbf{B}_{f}+\mathbf{B}_{b}\right)\right]
$$

where $\mathbf{B}_{f, b}=\mathbf{k} \times \mathbf{E}_{f, b} / \omega$ with $\mathbf{k} \| \mathbf{B}_{0}$ and $\mathbf{E}_{f, b} \perp \mathbf{B}_{0}$; $-e$ is electron charge; the subscripts $\mathrm{f}$ and $\mathrm{b}$ indicates forward and backward wave propagation, respectively, with respect to $\mathbf{B}_{0}$. Let $\phi$ be the gyration angle of $\mathbf{v}_{\perp}, \psi_{f, b}$ be the gyration angle of $\mathbf{B}_{f, b}$, and $\zeta_{f, b} \equiv \phi-\psi_{f, b}$ be the gyration angle between $\mathbf{v}_{\perp}$ and $\mathbf{B}_{f, b}$. We have

$$
\frac{d\left(\gamma v_{\|}\right)}{d t}=\frac{\Omega_{w}}{v_{\perp}}\left(\sin \zeta_{f}+\sin \zeta_{b}\right)
$$

\section{$\alpha=5$}

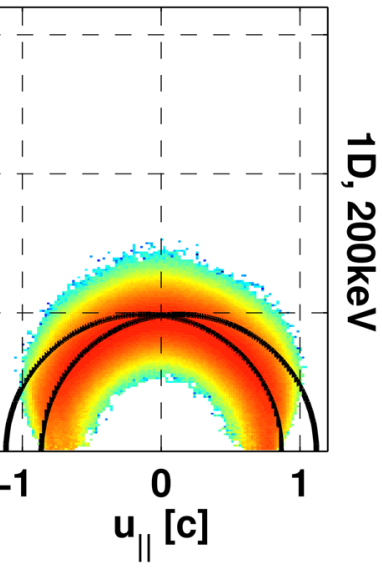

$$
\frac{d\left(\gamma v_{\perp}\right)}{d t}=\frac{\omega}{k} \Omega_{w}\left(\sin \zeta_{f}-\sin \zeta_{b}\right)-\Omega_{w} v_{\|}\left(\sin \zeta_{f}+\sin \zeta_{b}\right),
$$

$$
\begin{gathered}
\frac{d \gamma}{d t}=-\frac{e}{m_{e} c^{2}} \mathbf{v}_{\perp} \cdot\left(\mathbf{E}_{f}+\mathbf{E}_{b}\right)=\left(\frac{\omega}{k}\right)\left(\frac{\Omega_{w} v_{\perp}}{c^{2}}\right)\left(\sin \zeta_{f}-\sin \zeta_{b}\right), \\
\frac{d \phi}{d t}=\frac{\Omega_{w}}{\gamma v_{\perp}}\left[\cos \zeta_{f}\left(\frac{\omega}{k}-v_{\|}\right)+\cos \zeta_{b}\left(-\frac{\omega}{k}-v_{\|}\right)\right]+\frac{\Omega_{c e}}{\gamma}, \\
\theta_{f} \equiv \frac{d \zeta_{f}}{d t}=\frac{d \phi}{d t}-\left(\omega-k v_{\|}\right), \\
\theta_{b} \equiv \frac{d \zeta_{b}}{d t}=\frac{d \phi}{d t}-\left(\omega+k v_{\|}\right),
\end{gathered}
$$

where $\Omega_{w}=\Omega_{c e} B_{w} / B_{0}$ (see, e.g., Refs. 4 and 7). We have assumed the same wave amplitude, frequency and wave number for both forward and backward waves (two-wave case).

Fig. 8 shows the test-particle results with one wave, two waves and four waves (two forward waves and two backward waves) for the whistler-mode case corresponding to the simulation case with $\alpha=1$ and $\gamma_{0}=1.4$. The corresponding energetic electron distribution at the end of the simulation is also shown as color contours for comparison. The wave frequencies and vectors correspond to the most intensive waves in the $1 \mathrm{D}$ simulation. In the test-particle calculation, the wave amplitude is determined by the simulation result so that the total wave energy in the four-, two-, or one-wave case is equal to the wave peak energy in the 1D simulation.

Fig. 8(a) shows two one-wave cases in which electrons interact with a forward wave (purple) or a backward wave (blue), respectively. The electrons move along the diffusion curves with weak acceleration.

In Fig. 8(b), the two-wave case, electrons interact with one forward and one backward waves simultaneously. However, the superposition of all electron trajectories obtained from the test-particle calculation is slightly different from the simulation result as shown in Fig. 8(b). The test-particle trajectories form a thin "neck" while the 

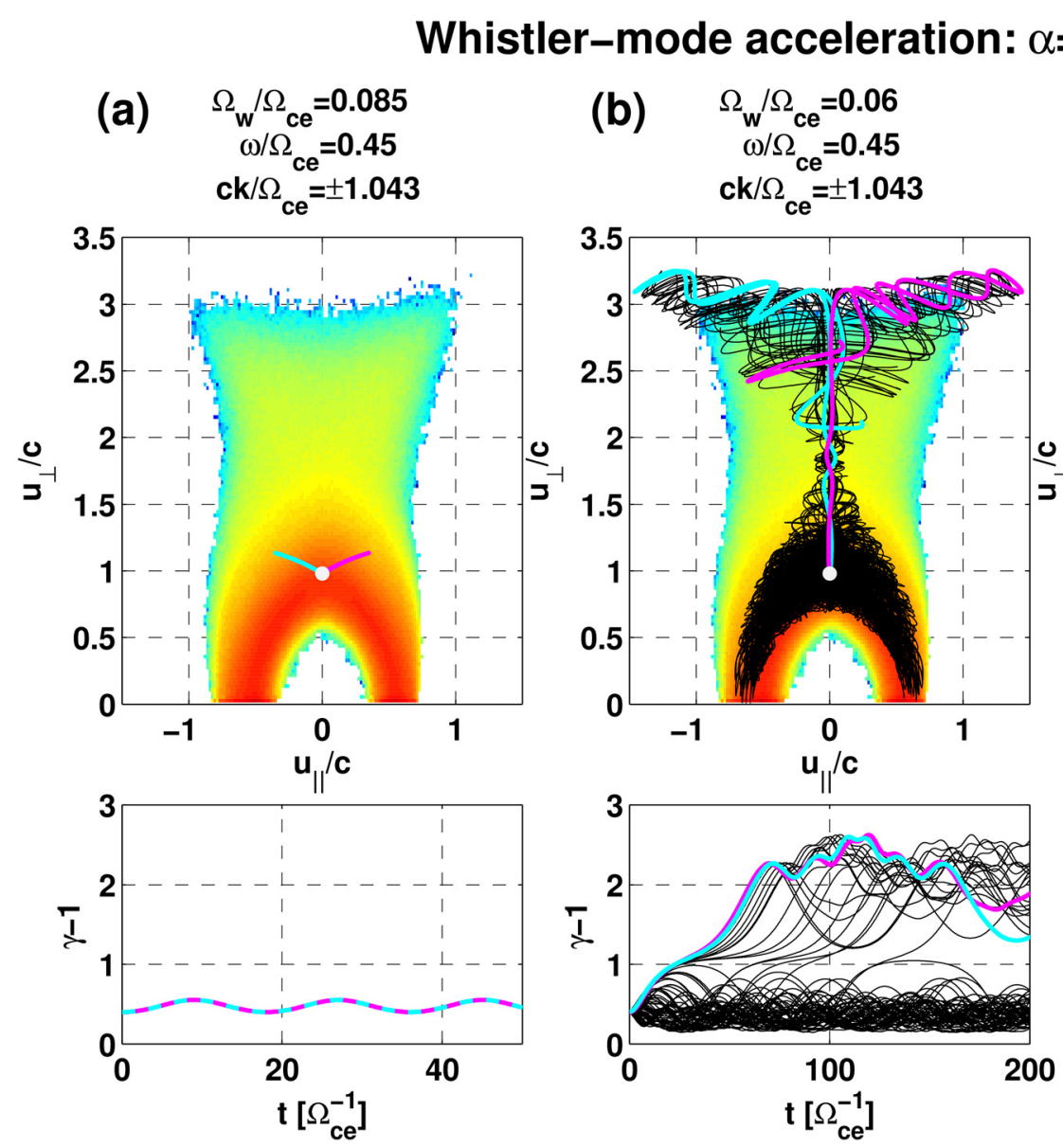
(c) $\Omega_{\mathrm{w}} / \Omega_{\mathrm{ce}}=\mathbf{0 . 0 4 2}$
$\omega / \Omega_{\mathrm{ce}}=[0.45,0.52]$
$\mathrm{ck} / \Omega_{\mathrm{ce}}=[ \pm 1.043, \pm 1.17]$
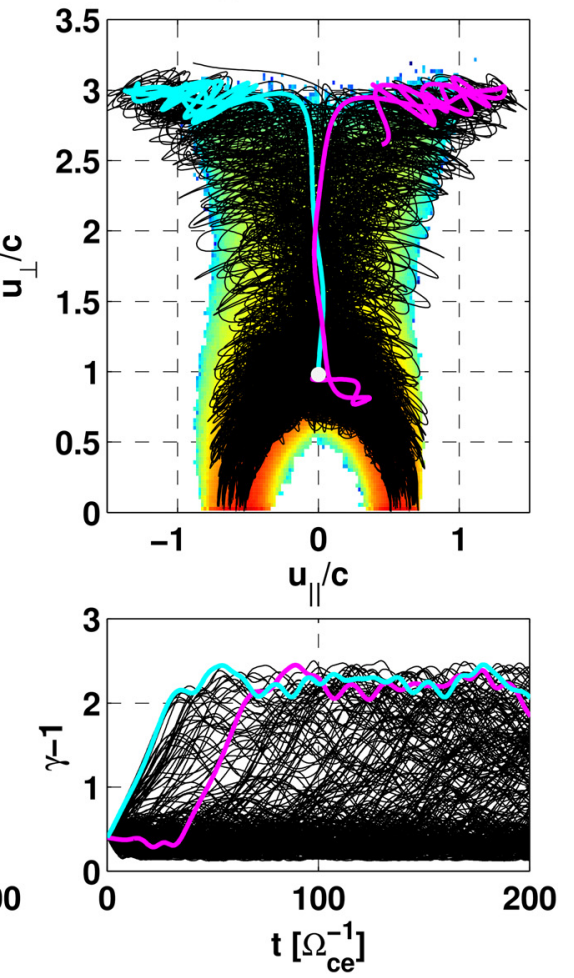

FIG. 8. Test-particle results with (a) one wave, (b) two waves, and (c) four waves. Electron trajectories in $u_{\perp}-u_{\|}$momentum space are plotted in the top panels, and kinetic energy time histories are shown in the bottom panels. The wave frequencies and wave numbers indicated at the top correspond to the most intensive waves in 1D simulations with $\alpha=1$ and $\gamma_{0}=1.4$. The corresponding energetic electron momentum distribution after wave energy saturation of the simulation $\left(t=512 \Omega_{c e}^{-1}\right)$ is also shown as color contours for comparison.

electron distribution from simulation has a thick "neck" in the distribution function.

In Fig. 8(c), the four-wave case, electrons interact with two forward and two backward waves simultaneously. The four-wave case further shows a more diffusive pattern which resembles the simulation result with a thick neck. The peak energy of accelerated electrons in the two-wave and four-wave cases can reach 6 times the initial kinetic energy.

In the two- and four-wave cases, the huge increment of electron energy is due to simultaneous acceleration by counter-propagating waves. ${ }^{7}$ During this stage, electrons are accelerated primarily in the perpendicular direction. After that, electron motion is dominated by waves in one direction, while the waves in the opposite direction just give a small modulation in energy and momentum. In Figs. 8(b) and 8(c), the purple curves correspond to electron motion in the later stage primarily dominated by forward waves, and the blue ones by the backward waves.

\section{TWO-WAVE RESONANT TRAPPING}

In Eq. (8), the acceleration is strong if the conditions $\zeta_{f}-\zeta_{b}=\pi \pm 2 n \pi$ ( $n$ is an integer) and $\zeta_{f}$ being within the acceleration phase are fulfilled. By assuming $\zeta_{f}-\zeta_{b}=$ $\pi \pm 2 n \pi$ and $v_{\|}=0$ in the initial condition, Eqs. (6), (10), and (11) give

$$
\begin{gathered}
\frac{d\left(\gamma v_{\|}\right)}{d t}=0, \\
\theta_{f}-\theta_{b}=2 k v_{\|}=0,
\end{gathered}
$$

which further ensures $v_{\|}=0$ and $\zeta_{f}-\zeta_{b}=\pi \pm 2 n \pi$ all the time. Based on the assumption that $\zeta_{f}-\zeta_{b}=\pi \pm 2 n \pi$ and $v_{\|}=0$, the governing equations for ideal two-wave resonance can be simplified as

$$
\begin{gathered}
\frac{d u_{\perp}}{d t}=2 \frac{\omega}{k} \Omega_{w} \sin \zeta_{f}, \\
\frac{d \gamma}{d t}=2 \frac{\omega}{k} \frac{\Omega_{w} u_{\perp}}{\gamma c^{2}} \sin \zeta_{f}, \\
\theta_{f} \equiv 2 \frac{\omega}{k} \frac{\Omega_{w}}{u_{\perp}} \cos \zeta_{f}+\frac{\Omega_{c e}}{\gamma}-\omega .
\end{gathered}
$$

We solve the governing equations numerically and obtain particle trajectories in $u_{\perp}-\zeta_{f}$ and $\theta_{f}-\zeta_{f}$ phase space and 
time histories of kinetic energy as shown in Fig. 9. The wave frequencies and wave numbers correspond to the most intensive waves in the 1D simulations. Two-wave resonant trapping occurs and can lead to a strong acceleration of electrons, and the blue curves correspond to maximum twowave acceleration.

The straight line in the $u_{\perp}-\zeta_{f}$ plot corresponds to the initial electron positions with $u_{\perp} \approx 0.98 c\left(\gamma_{0}=1.4\right)$ and different phase $\zeta_{f}$. The section with red (gray) color is for trapped (untrapped) electrons. In $\theta_{f}-\zeta_{f}$ plot, the corresponding curves of the initial positions for trapped and untrapped electrons with $\gamma_{0}=1.4$ are also drawn. The horizontal dashed line with $u_{\perp}=u_{\perp c}$ in the $u_{\perp}-\zeta_{f}$ plot indicates the vertical center of trapping zone, where $u_{\perp c}$ is defined by $\theta_{f}=0$ with $\zeta_{f}=\pi$. In the case of $\alpha=0.33$, most electrons are trapped, but the maximum energy increment from $\gamma_{0}=1.4$ is only $\Delta \gamma \approx 0.5$. In the case of $\alpha=1$, although the number of trapped electrons is lower, the energy increment of all these trapped electrons can reach $\Delta \gamma \approx 2$ from $\gamma_{0}=1.4$. In the case of $\alpha=5$, no electron of $\gamma_{0}=1.4$ is trapped by two-wave resonance, and hence acceleration in this case is weak.

By differentiating Eq. (16) in time, we can obtain the second order resonance condition

$$
\begin{aligned}
& \frac{1}{\Omega_{c e}^{2}} \frac{d \theta_{f}^{2}}{d \zeta_{f}}+4\left(\frac{\omega}{c k} \frac{\Omega_{w}}{\Omega_{c e}}\right)\left(\frac{c}{u_{\perp}}\right) \\
& \quad \times\left(4 \frac{\omega}{k} \frac{\Omega_{w}}{\Omega_{c e} u_{\perp}} \cos \zeta_{f}-\frac{\omega}{\Omega_{c e}}+\frac{1}{\gamma}+\frac{u_{\perp}^{2}}{\gamma^{3} c^{2}}\right) \sin \zeta_{f}=0 .
\end{aligned}
$$
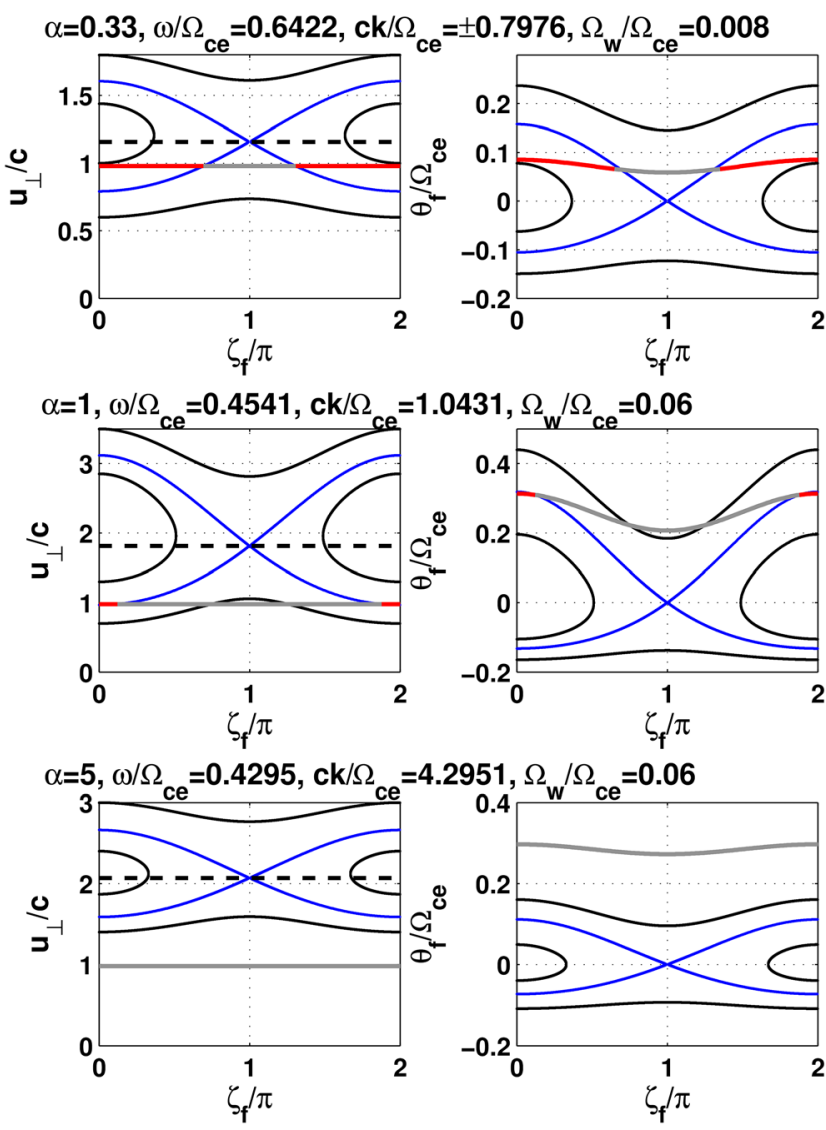

For small wave amplitude, the term with $\Omega_{w} \cos \zeta_{f}$ can be ignored, and we define the trapping frequency as

$$
\frac{\omega_{t r}^{2}}{\Omega_{c e}^{2}} \equiv 4\left(\frac{\omega}{c k} \frac{\Omega_{w}}{\Omega_{c e}}\right)\left(\frac{c}{u_{\perp}}\right)\left(-\frac{\omega}{\Omega_{c e}}+\frac{1}{\gamma}+\frac{u_{\perp}^{2}}{\gamma^{3} c^{2}}\right) .
$$

Equation (17) further indicates that the trajectories in phase space should be pendulum-like. The factor $\omega \Omega_{w} / c k \Omega_{c e}$ in Eq. (18) controls the vertical width of trapping zone, and wave frequency determines the vertical center with $\left(-\omega / \Omega_{c e}\right)$ in $u_{\perp}-\zeta_{f}$ phase space. For $\alpha=0.33$, we have $\omega / \Omega_{c e} \approx 0.64$, $\omega / c k \approx 0.8, \Omega_{w} / \Omega_{c e}=0.008$, and $\omega \Omega_{w} / c k \Omega_{c e} \approx 0.006$. For $\alpha=1$, we have $\omega / \Omega_{c e} \approx 0.45, \omega / c k \approx 0.44, \quad \Omega_{w} / \Omega_{c e}$ $=0.06$, and $\omega \Omega_{w} / c k \Omega_{c e} \approx 0.026$. For $\alpha=5$, we have $\omega / \Omega_{c e} \approx 0.43, \omega / c k \approx 0.1, \Omega_{w} / \Omega_{c e}=0.06$, and $\omega \Omega_{w} / c k \Omega_{c e}$ $\approx 0.006$. In Fig. 9, among the three cases, the maximum vertical width of trapping zone occurs in the case of $\alpha=1$ due to largest $\omega \Omega_{w} / c k \Omega_{c e}$, leading to maximum kinetic energy increment for trapped electrons. The highest vertical center of trapping zone in $u_{\perp}-\zeta_{f}$ phase space occurs in the case of $\alpha=5$ due to low wave frequency, and electrons with initial energy $\gamma_{0}=1.4$ are all untrapped. There seems to exist a threshold of $\Omega_{w} / \Omega_{c e}$ for two-wave resonant trapping. If $\omega \Omega_{w} / c k \Omega_{c e}$ is smaller, the trapping zone is thinner, and the maximum energy increment of two-wave acceleration is lower. If $\omega / \Omega_{c e}$ is lower, the trapping zone shifts upward in $u_{\perp}-\zeta_{f}$ phase space, leading to a higher threshold of $\Omega_{w} / \Omega_{c e}$ for two-wave resonant trapping.

Fig. 10 shows maximum energy gain of accelerated electrons as a function of wave amplitude for one- and
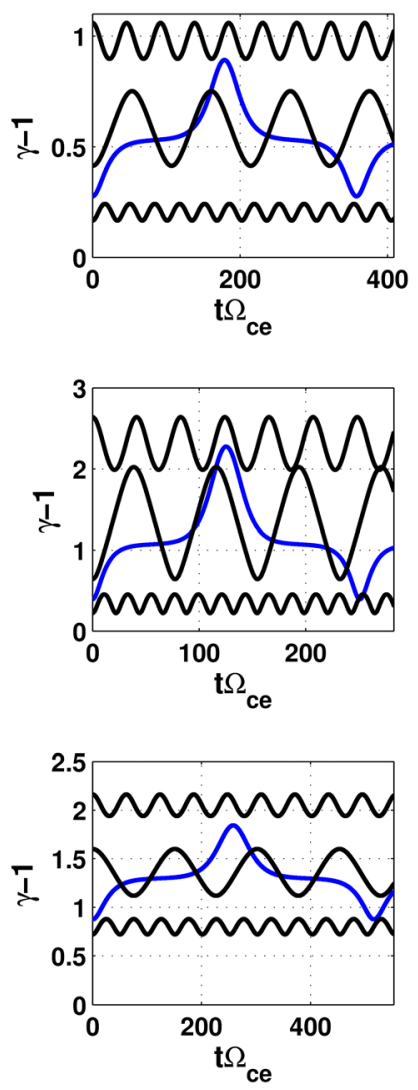

FIG. 9. Test-particle results of twowave cases corresponding to $\alpha=0.33$, 1 , and 5. It is assumed that $v_{\|}=0$ and $\zeta_{b}=\zeta_{f}+\pi$. Blue curves correspond to the maximum acceleration due to twowave resonant trapping. The red and gray lines in $u_{\perp}-\zeta_{f}$ and $\theta_{f}-\zeta_{f}$ plots indicate initial positions of electrons with $u_{\perp} \approx 0.98\left(\gamma_{0}=1.4\right)$. The section with red (gray) color is for trapped (untrapped) electrons. The horizontal dashed line in $u_{\perp}-\zeta_{f}$ plot indicates the vertical center of the trapping zone. 

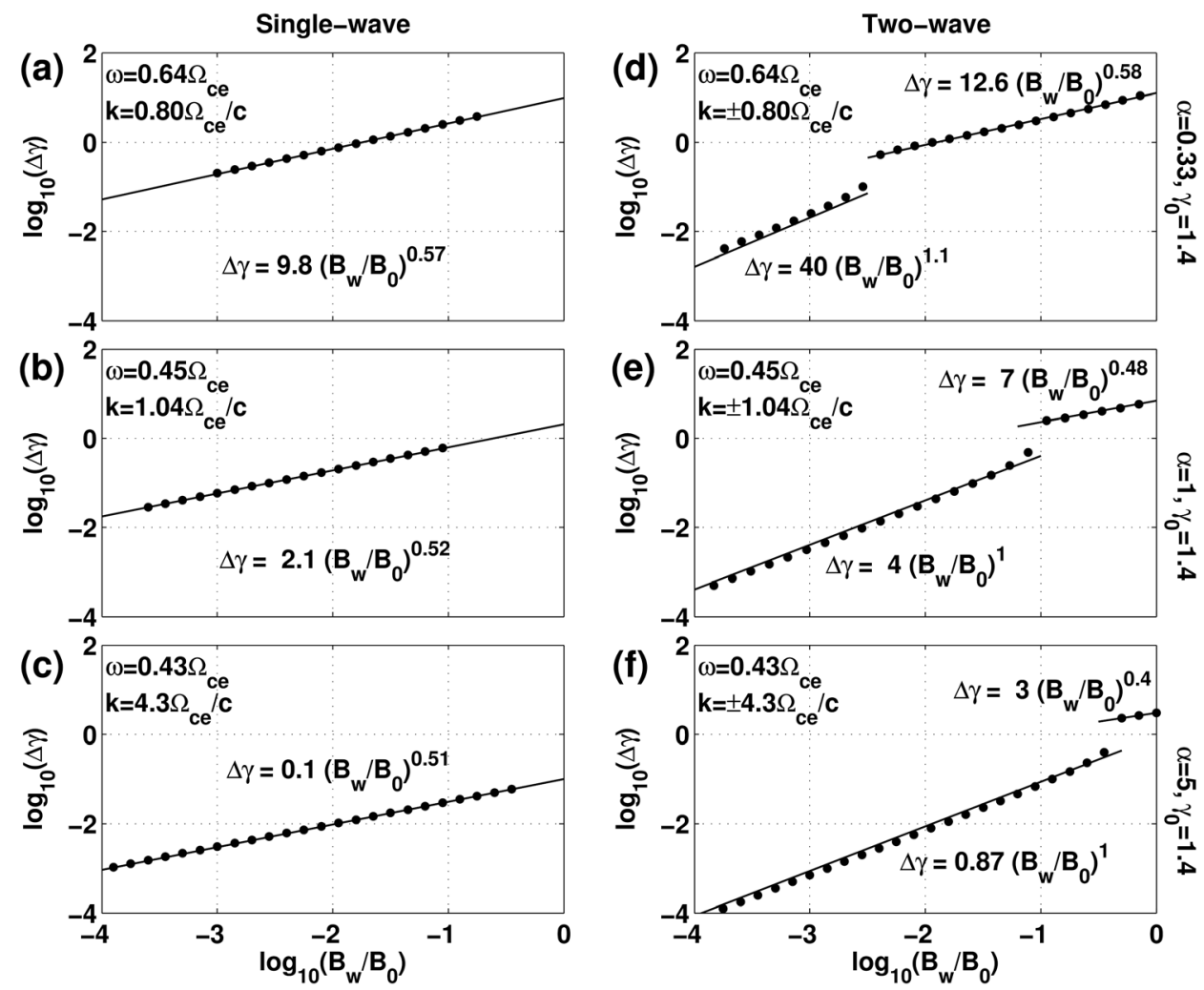

FIG. 10. Maximum energy increment (dots) of accelerated electrons as a function of wave amplitude for oneand two-wave cases with $\gamma_{0}=1.4$ and $\alpha=0.33,1$, and 5 obtained from testparticle calculations. The best fitting lines are also plotted. The initial condition for electrons in the one-wave cases is $\gamma_{0}=1.4$ with randomized $u_{\downarrow}$ $u_{\|}$and $\zeta ; \gamma_{0}=1.4$ with $u_{\perp}=0.98 c$ $u_{\|}=0$ and randomized $\zeta_{f}$ and $\zeta_{b}$ in the two-wave cases. two-wave cases with $\gamma_{0}=1.4$ and $\alpha=0.33,1$, and 5 obtained from test-particle calculations. Note that here we do not assume $v_{\|}=0$ and $\zeta_{f}-\zeta_{b}=\pi \pm 2 n \pi$, and the initial conditions of $\zeta_{f}$ and $\zeta_{b}$ are randomly given. There exists a gap in wave amplitude for strong two-wave resonant acceleration. The acceleration is weak if wave amplitude is lower than the threshold.

In the one-wave cases, electrons always move along diffusion curves, which is determined by the wave phase velocity. Diffusion curves of higher phase velocity can cross through higher energy region in the velocity or momentum space as the case of $\alpha=0.33$ shown in Fig. 7. Strong acceleration occurs only if the wave has high phase velocity and large amplitude.

In the test-particle calculations, the two-wave resonant acceleration is reversible as shown in Fig. 9. However, it can be irreversible if Doppler effect comes in at a later stage.
This can be achieved if $d v_{\|} / d t \neq 0$ due to a small difference in wave frequency, wave amplitude or phase between $\zeta_{f}$ and $\zeta_{b}+\pi \pm 2 n \pi$. We consider a single electron with the initial condition, $\zeta_{f}-\zeta_{b}=0.99 \pi \pm 2 n \pi$ and $\zeta_{f}=0$, and solve the full set of equations of electron motion [Eqs. (6)-(11)], and the result is shown in Fig. 11. The electron is initially trapped by two-wave resonance as the red curves in Fig. 11. The two-wave trapping process can be identified by the red curve in Fig. 11(a). Due to $d v_{\|} / d t \neq 0$, we have Doppler effect or $v_{\|} \neq 0$ at a later stage. The electron is de-trapped by the Doppler effect and then trapped again by one of the two counter-propagating waves, ${ }^{1}$ as shown by the blue curves in Fig. 11. The other wave propagating in opposite direction just gives a small modulation in electron energy and momentum during one-wave resonant trapping as shown in Figs. 11(c) and 11(d). The one-wave resonant trapping can further accelerate electrons to a higher energy level at the

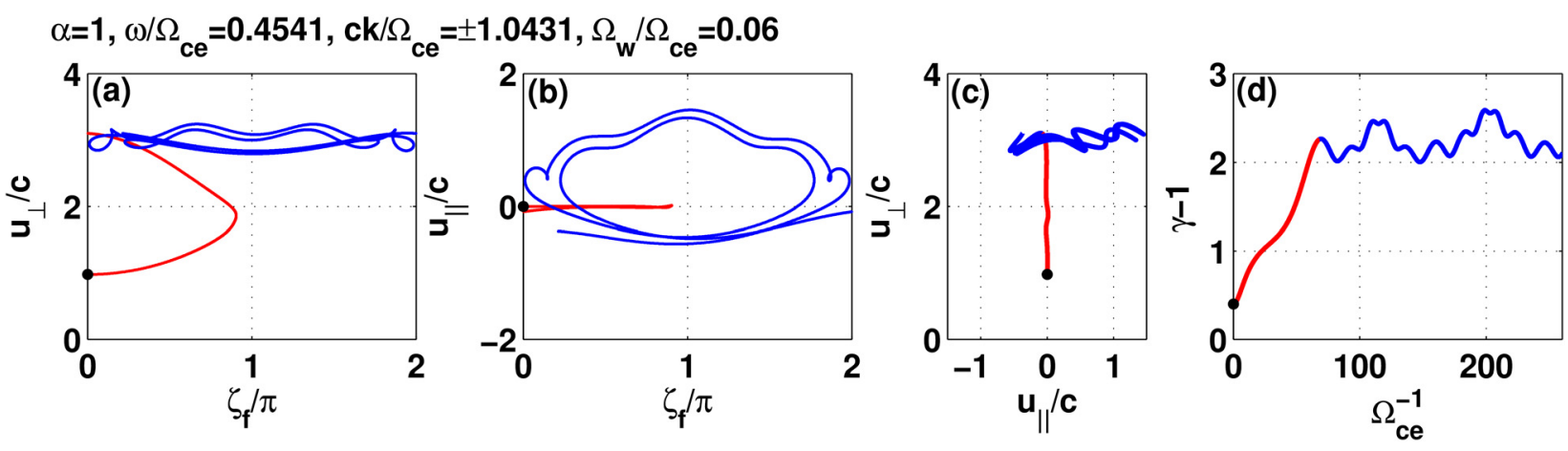

FIG. 11. Test-particle results of a single electron in the two-wave case corresponding to $\alpha=1$ with initial conditions $\zeta_{f}=\zeta_{b}+0.99 \pi$ and $\zeta_{f}=0$. Red parts of the electron trajectory curves are associated mainly with the two-wave resonant trapping at the early stage; blue parts are associated mainly with one-wave resonant trapping at the late stage. Black dots indicate initial positions. 


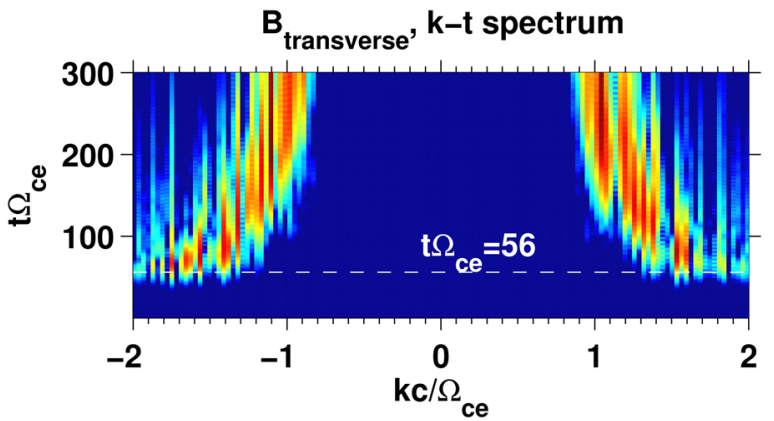

FIG. 12. $k-t$ power spectrum for transverse magnetic field of the case with $\alpha=1$ and $\gamma_{0}=1.4$. At $t \Omega_{c e}=56$ (dashed line), the wave number of the most intensive whistler-mode waves is $c k_{\text {peak }} / \Omega_{c e} \approx 1.6$, corresponding to the peak wavelength $\lambda_{\text {peak }} \approx 4 c / \Omega_{c e}$.
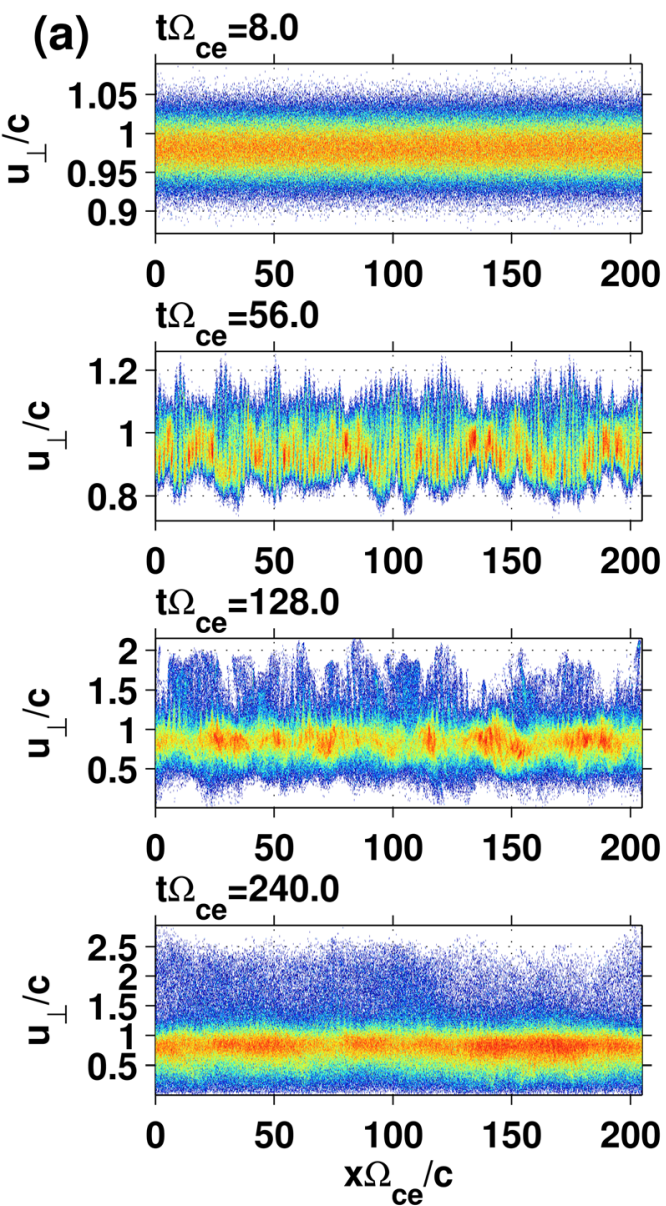

(b) $t \Omega_{c e}=56.0$

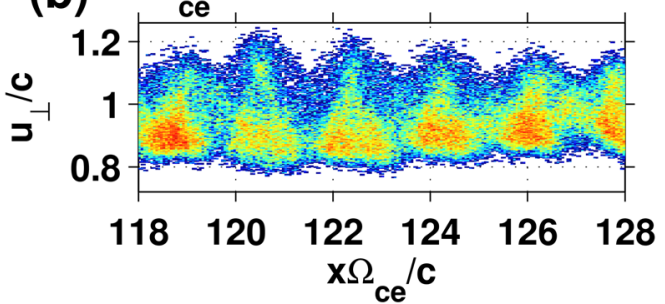

FIG. 13. (a) Evolution of energetic electron distribution in $u_{\perp}-x$ phase space of the case with $\alpha=1$ and $\gamma_{0}=1.4$ at different times. (b) An enlargement of the $u_{\perp}-x$ plot at $t \Omega_{c e}=56$, which shows strong two-wave resonant acceleration with wavelength $\lambda_{a c c} \approx 2 c / \Omega_{c e}$. later stage. In Fig. 11, the energy gain of two-wave acceleration is $\Delta \gamma \approx 1.9$, and that of one-wave acceleration at a later stage is $\Delta \gamma \approx 0.3$. The total energy gain is about 5.5 times the initial kinetic energy or $\Delta \gamma \approx 2.2=5.5\left(\gamma_{0}-1\right)$.

The phase difference between $\zeta_{f}$ and $\zeta_{b}$ can be written as

$$
\begin{aligned}
\zeta_{f}-\zeta_{b} & =\left(\phi-\psi_{f}\right)-\left(\phi-\psi_{b}\right)=\psi_{f}-\psi_{b} \\
& =\left(\omega t-k x+\psi_{f 0}\right)-\left(\omega t+k x+\psi_{b 0}\right) \\
& =-2 k x+\left(\psi_{f 0}-\psi_{b 0}\right),
\end{aligned}
$$

where $\psi_{f}$ and $\psi_{b}$ are the phases of forward and backward waves, respectively; $\psi_{f 0}$ and $\psi_{b 0}$ are the phase constants of forward and backward waves, respectively. There is no time dependency on the phase difference. At $\zeta_{f}-\zeta_{b}=\pi \pm 2 n \pi$, strong two-wave resonant acceleration in the perpendicular direction occurs. Therefore the efficient acceleration takes place at a spatial interval corresponding to a wave number of $2 k$. We examine the particle simulation results of the case with $\alpha=1$ and $\gamma_{0}=1.4$. At $t \Omega_{c e}=56$, the wave number of the most intensive waves is $c k_{\text {peak }} / \Omega_{c e} \approx 1.6$, corresponding to peak wavelength $\lambda_{\text {peak }} \approx 4 c / \Omega_{c e}$, as shown by Fig. 12. In Fig. 13(a), the evolution of electron distribution in $u_{\perp}-x$ phase space shows clearly the wavy structure before $t \Omega_{c e}=100$. As $-2 k x+\left(\psi_{f 0}-\psi_{b 0}\right)$ varies in space, strong two-wave resonant acceleration occurs at an interval of $\lambda_{\text {acc }} \approx 2 c / \Omega_{c e} \approx 0.5 \lambda_{\text {peak }}$ as shown in Fig. 13(b). At the late stage, most electrons are accelerated in the parallel direction, and the wavy structure of trapped electrons in $u_{\perp}-x$ phase space becomes vague.

\section{DISCUSSION AND SUMMARY}

The electron ring distribution can lead to amplification of parallel Z- and whistler-mode waves. Intensive interactions between these waves and ring electrons lead to acceleration and deceleration of electrons.

For ideal two-wave resonant trapping, the governing equation can be simplified to a pendulum-like equation base on the assumptions, $\zeta_{f}-\zeta_{b}=\pi \pm 2 n \pi$ and $v_{\|}=0$. The vertical width of trapping zone is proportional to the value of $\omega \Omega_{w} / c k \Omega_{c e}$, which affects the maximum energy gain of trapped electrons. The vertical center $\left(u_{\perp c}\right)$ of the trapping zone in $u_{\perp}-\zeta_{f}$ phase space is related to $\left(-\omega / \Omega_{c e}\right)$, and hence a higher frequency can lower the trapping threshold of wave amplitude $\Omega_{w} / \Omega_{c e}$.

The ideal two-wave resonant acceleration is a reversible process as shown in the test-particle results. However, if initially there exists a small difference between $\zeta_{f}$ and $\zeta_{b}+$ $\pi \pm 2 n \pi$ or in wave frequency or wave amplitude, the electrons can be de-trapped at a later stage due to the Doppler effect introduced by $d v_{\|} / d t \neq 0$. These electrons can be further trapped at a later stage by one-wave resonance and accelerated to a slightly higher energy.

There exist three types of electron acceleration and diffusion. (i) For Z-mode waves with high phase velocity $\left(v_{p h} \approx 0.8 c\right)$, the electron acceleration and diffusion in momentum space can be directly related to the one-wave resonant diffusion. (ii) For whistler-mode waves with medium 
phase velocity $\left(v_{p h} \approx 0.4 c\right)$, two-wave resonant acceleration is needed and very effective, leading to a strong energization of electrons. (iii) For whistler-mode waves with low phase velocity $\left(v_{p h} \approx 0.1 c\right)$, the threshold of wave amplitude for two-wave acceleration is high. If no electron is trapped by two-wave resonance, electrons only undergo pitch-angle scattering with little energy variation.

In the particle simulations and test-particle calculations, the peak energy of accelerated electrons can reach 2-8 times the initial kinetic energy. The initial kinetic energy in our simulations ranges from 100 to $500 \mathrm{keV}$, while the final peak energy can reach 200 to $3000 \mathrm{keV}$.

The main results can be summarized as follows.

(a) Simultaneous acceleration of electrons by counterpropagating waves can be very effective while acceleration in one-wave cases is due to electron movement along the diffusion curve.

(b) For ideal two-wave resonant trapping, higher $\omega \Omega_{w} / c k \Omega_{c e}$ leads to a thicker trapping zone, and the maximum energy increment is higher as in the case of $\alpha=1$. On the other hand, lower wave frequency shifts the trapping zone upward with $\left(-\omega / \Omega_{c e}\right)$ in $u_{\perp}-\zeta_{f}$ phase space, leading to a higher threshold of $\Omega_{w} / \Omega_{c e}$ as in the case of $\alpha=5$. The acceleration is very weak if $\Omega_{w} / \Omega_{c e}$ is below the threshold.

(c) There exist three types of electron acceleration and diffusion: (i) dominant one-wave resonant trapping with high wave phase velocity, leading to a sharp X-like electron distribution in momentum space, as in the case of $\alpha=0.33$; (ii) dominant two-wave resonant trapping with medium wave phase velocity, leading to an electron distribution with long "neck," as in the case of $\alpha=1$, and (iii) no trapped electron in the acceleration phase with low wave phase velocity, leading to a shelllike distribution, as in the case of $\alpha=5$.

(d) The ideal two-wave resonant acceleration is a reversible process. However, electrons can be de-trapped at a later stage due to the Doppler effect introduced by $d v_{\|} / d t \neq 0$.

(e) The efficient two-wave resonant acceleration takes place at a spatial interval $\lambda_{a c c}$, which equals to half of the peak wavelength $\lambda_{\text {peak }}$ of the most intensive wave mode $\left(\lambda_{\text {acc }} \approx 0.5 \lambda_{\text {peak }}\right)$.

(f) In the 1D and 2D particle simulations with $\alpha=0.33$ and $\gamma_{0}=1.2,1.4$, and 2 , the peak energy of accelerated electrons is about $3 \sim 4$ times the initial kinetic energy; with $\alpha=1$, it can reach $6 \sim 8$ times the initial kinetic energy; with $\alpha=5$, it can reach only $2 \sim 3$ times the initial kinetic energy.

\section{ACKNOWLEDGMENTS}

This work was supported by Grant-in-Aid 23340147 of the Ministry of Education, Science, Sports and Culture of Japan, by the National Science Council in Taiwan (NSC 101-2628-M-001-007-MY3), and by the Center for Computational Geophysics of NCU in Taiwan (CCG Contribution No. NCU-CCG102-0004).

${ }^{1}$ Y. Katoh and Y. Omura, J. Geophys. Res. 109, A12214, doi:10.1029/2004JA010654 (2004); Y. Omura, N. Furuya, and D. Summers, ibid. 112, A06236, doi:10.1029/2006JA012243 (2007); Y. Katoh and Y. Omura, Geophys. Res. Lett. 34, L13102, doi:10.1029/2007GL029758 (2007); D. Summers and Y. Omura, ibid. 34, L24205, doi:10.1029/2007GL032226 (2007); N. Furuya, Y. Omura, and D. Summers, J. Geophys. Res. 113, A04224, doi:10.1029/2007JA012478 (2008); Y. Katoh, Y. Omura, and D. Summers, Ann. Geophys. 26, 3451, doi:10.5194/angeo-26-3451-2008 (2008); M. Hikishima, Y. Omura, and D. Summers, J. Geophys. Res. 115, A12246, doi:10.1029/2010JA015860 (2010).

${ }^{2}$ D. Summers, B. Ni, and N. P. Meredith, J. Geophys. Res. 112, A04207, doi:10.1029/2006JA011993 (2007); A. Varotsou, D. Boscher, S. Bourdarie, R. B. Horne, N. P. Meredith, S. A. Glauert, and R. H. Friedel, ibid. 113, A12212, doi:10.1029/2007JA012862 (2008); Z. Su, F. Xiao, H Zheng, and S. Wang, ibid. 116, A04205, doi:10.1029/2010JA016228 (2011).

${ }^{3}$ Y. Omura and Q. Zhao, J. Geophys. Res. 117, A08227, doi:10.1029/2012JA017943 (2012); Y. Omura and Q. Zhao, J. Geophys. Res. [Space Phys.] 118, 5008, doi:10.1002/jgra.50477 (2013).

${ }^{4}$ Y. Omura and D. Summers, J. Geophys. Res. 111, A09222, doi:10.1029/2006JA011600 (2006).

${ }^{5}$ C. S. Wu and L. C. Lee, Astrophys. J. 230, 621 (1979); L. C. Lee and C. S. Wu, Phys. Fluids 23, 1348 (1980).

${ }^{6}$ K. H. Lee, Y. Omura, L. C. Lee, and C. S. Wu, Phys. Rev. Lett. 103, 105101 (2009); K. H. Lee, Y. Omura, and L. C. Lee, Phys. Plasmas 18 092110 (2011)

${ }^{7}$ K. H. Lee, Y. Omura, and L. C. Lee, Phys. Plasmas 19, 122902 (2012).

${ }^{8}$ P. H. Yoon, C. B. Wang, and C. S. Wu, Phys. Plasmas 14, 022901 (2007).

${ }^{9}$ C. S. Wu, J. Geophys. Res. 89, 8857, doi:10.1029/JA089iA10p08857 (1984); A. O. Benz and G. Thejappa, Astron. Astrophys. 202, 267 (1988); S. Kainer and R. J. MacDowall, J. Geophys. Res. 101, 495, doi:10.1029/95JA02026 (1996).

${ }^{10}$ S. M. White, D. B. Melrose, and G. A. Dulk, Astrophys. J. 308, 424 (1986); C. S. Wu, C. B. Wang, G. C. Zhou, S. Wang, and P. H. Yoon, ibid. 621, 1129 (2005)

${ }^{11}$ H. P. Freund, H. K. Wong, C. S. Wu, and M. J. Xu, Phys. Fluids 26, 2263 (1983); Y. P. Chen, G. C. Zhou, P. H. Yoon, and C. S. Wu, Phys. Plasmas 9, 2816 (2002)

${ }^{12}$ Y. Omura, "One-dimensional electromagnetic particle code: KEMPO1," in Advanced Methods for Space Simulations, edited by H. Usui and Y. Omura (Terra Sci., Tokyo, 2007), pp. 1-21.

${ }^{13}$ A. D. M. Walker, Plasma Waves in the Magnetosphere (Springer-Verlag, New York, 1993), pp. 151-186; D. Summers, R. M. Thorne, and F. Xiao, J. Geophys. Res. 103, 20487, doi:10.1029/98JA01740 (1998). 